\title{
Formation mechanism of microcrystalline spherical graphite particles in solidified nickel
}

\author{
Mao-Hua Teng *, Chung-I Hsiao, Yuan-Lung Hsiao \\ Department of Geosciences, National Taiwan University, Taipei 106, Taiwan
}

\section{A R T I C L E I N F O}

Available online 4 September 2008

\section{Keywords:}

Synthetic diamond

Graphite

Metastable phases

Arc-discharge

\begin{abstract}
A B S T R A C T
Nickel is a well known catalyst that can readily transform carbon into graphite at high temperature. In our designed arc-discharge experiments, diamond easily dissolved in melted nickel and became small graphitic flakes. After the melted nickel had cooled and solidified, there were many microcrystalline-sized spherical graphite particles found on the nickel grain boundaries, surprisingly. It is interesting that only at the hotter area would the spherical graphite be found. This leads to our proposed forming mechanism: the spherical graphite particles were formed during the solidification of nickel. Because the temperature and solubility are high at the hotter area (zone A), the crystallization and growth of graphite became transport-controlled, instead of surface-controlled, and therefore the most common shape of the particles was spherical. This phenomenon, as far as we know, has not been previously observed.
\end{abstract}

(C) 2008 Elsevier B.V. All rights reserved.

\section{Introduction}

Nickel, like iron and cobalt, is a well known catalyst that can readily transform carbon into graphite at high temperature [1-2]. The original carbon could be amorphous carbon or diamond [3-4]. In this work, we dissolved synthetic diamond particles in melted nickel. After the nickel had cooled and solidified, we found not only flakes of graphite but also many microcrystalline "spherical" graphite particles in the nickel block. The purpose of this work is to investigate the phenomenon and to propose a possible forming mechanism of these spherical graphite particles. As far as we know, this phenomenon has not been observed in a Ni-C system nor reported hitherto.

\section{Experimental}

An arc-discharge setup was used to quickly melt the nickel and dissolve the diamond particles (Fig. 1(a)). The setup not only put a lot of heat into the nickel in a relatively short time, but also established a temperature gradient inside the nickel, as shown in Fig. 1(b). Zone A is the closest portion of the nickel to the arc-discharge, zone $C$ is the farthest, and zone B is in between zones $A$ and $C$. The $99.9 \%$ pure nickel pieces and diamond particles, about $100 \mu \mathrm{m}$ in diameter, were put into a graphite crucible with a weight ratio of about 10:1. The crucible also served as the anode of the setup. The arc-discharge ran at $100 \mathrm{amp}$

\footnotetext{
* Corresponding author. Tel.: +886227396359.

E-mail address: mhteng@ntu.edu.tw (M.-H. Teng).
}

and $20 \mathrm{~V}$ in a 300 Torr helium atmosphere and lasted for 5 to over 30 min. Once the arc-discharge was being shut down, the nickel block cooled rapidly and solidified in seconds. Then the nickel block could be removed, cut, polished, and observed under SEM.

\section{Results and discussion}

No diamond particles could be found inside the nickel block after just $5 \mathrm{~min}$ in the arc-discharge experiment. However, this may not necessarily mean that the metastable diamond had all transformed into the stable phase graphite during the experiments. Some large amount of carbon atoms may dissolve in the melted nickel, especially at the high temperature zone A. Based on the study of graphite encapsulated metal (GEM) nanoparticles [5-7], when the liquid-like metal-carbon mixture are quenched in an evaporation-condensation process, many nanocrystalline turbostratic graphitic sheets become crystallized and encapsulate the metal particles. This may indicate that in our experiment at zone A, numerous nanocrystalline graphitic sheets may co-exist and mix with carbon-saturated nickel melts. Because the temperatures are lower at zones B and $\mathrm{C}$, the carbon concentration should be lower, accordingly.

Although at the center of an arc-discharge the temperature could reach several thousands degree Celsius, the maximum temperature inside the melted nickel pool could still be around $1500{ }^{\circ} \mathrm{C}$ due to the evaporation process on the surface of the melting nickel pool, and the temperature gradually decreases from zone A to zone C (Fig. 1(b)), where the temperature may be just a little below the melting point of nickel (i.e. $1453^{\circ} \mathrm{C}$ ). The temperature gradient has a dramatic effect on the distribution of carbon. At zone A, with higher temperature and higher carbon solubility, no large graphite flakes could be found in the 


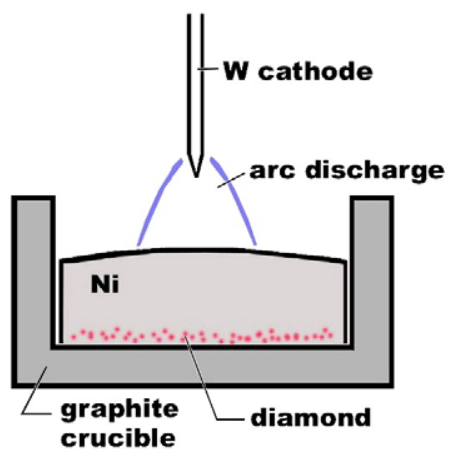

(a) temperature gradient $A>B>C$

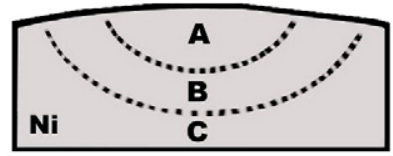

(b)
Fig. 1. (a) The schematic of an arc-discharge setup. The diamond particles dissolved in melted nickel in the first 5 min period. (b) The temperature gradient was established during arc-discharge, where the temperature at zone $\mathrm{A}$ is higher than at zone $\mathrm{B}$, and zone $C$ is at lowest temperature.

solidified samples, only agglomerated small spherical-like graphite particles (Fig. 2); at zone C, on the other hand, with lower temperature and lower carbon solubility, many large graphite flakes near the bottom of the nickel block could be found (Fig. 3). At zone B, only small graphite flakes on the grain boundaries of nickel were found. In other words, from zone $A$ to zone $C$ the mechanism of the crystallization and growth of graphite was gradually changed because of the temperature gradient.

Fig. 2 is a two-dimensional cross-section of a three dimensional arrangement of many microcrystalline graphite particles sitting on the grain boundaries of solidified nickel. These graphite particles are not similar to the individual spherical graphite particles found in ductile cast iron [8-9]; instead, they are agglomerated in certain patterns, showing the grain boundaries of nickel grains. The forming mechanism is also different; unlike ductile cast iron where several additives must be added and which must go through a long annealing process, the spherical graphite particles found in zone A were formed directly from a quickly solidified nickel. As mentioned earlier, there are numerous nanocrystalline graphitic sheets and saturated carbon atoms in zone A, and may be quenched with very high value of carbon supersaturation. Note that some of the nickel boundaries do not have graphite particles on them; this may indicate that at the early stage of nickel grain growth, when the temperature is still relatively high, the growing grains bump into each other and "squeeze" the spherical graphite particles into the surrounding not yet solidified areas. Some parts of Fig. 2, the upper

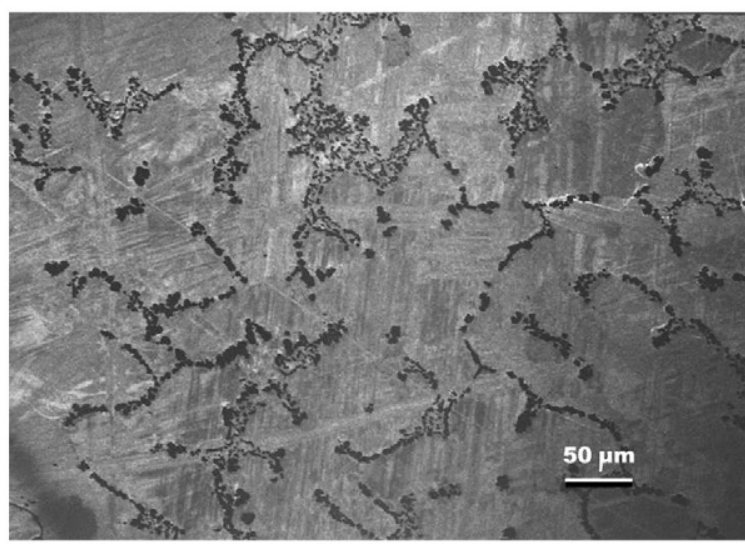

Fig. 2. SEM image of many microcrystalline spherical graphite particles found at the hotter zone A (see Fig. 1(b)) in a solidified 5 min arc-discharge nickel block.

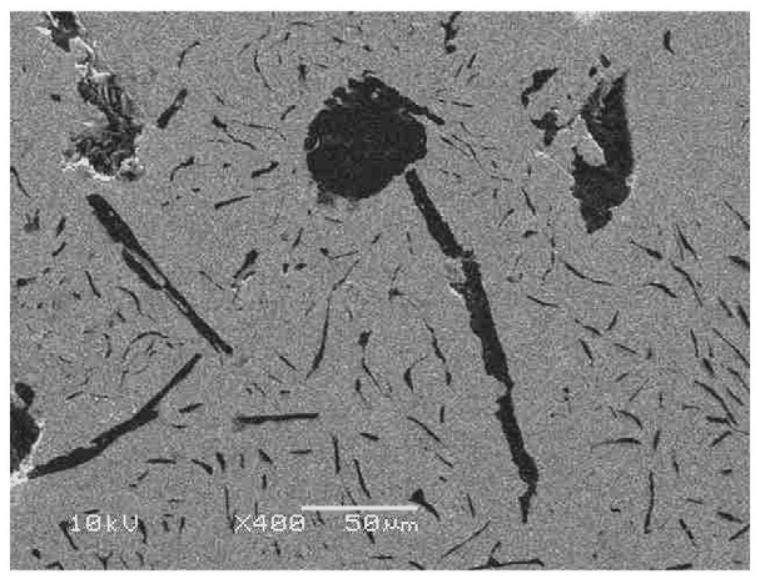

Fig. 3. SEM image of many large platy graphite particles found at the cooler zone C (see Fig. 1(b)) in a solidified 5 min arc-discharge nickel block.

middle area, show the cross-section of these portions that solidified in the final stage, and were densely occupied by these spherical graphite particles.

Fig. 4 is a transition from zone A on the left to zone B on the righthand side of the SEM micrograph. The graphite particles in zone B, though still apparently located on the nickel grain boundaries, are much larger and exhibit irregular shapes. Note that no large plateshaped graphite could be found.

Fig. 5 is the cross-section of zone A, but after more than 10 min of arc-discharge. Note that the spherical graphite particles disappeared, and were replaced by large graphitic flakes. In a sample after $2 \mathrm{~h}$ of arc-discharge, in the same area (zone A) one cannot find any visible graphite (Fig. 6). As a result, the temperature gradient during the runs makes zone C a "collector" that all the carbon atoms or small graphitic flakes will tend to diffuse and stay in the cooler zone $C$ during a twohour experiment.

In their experiments on the crystallization of a mineral plagioclase from plagioclase melts, Lofgren et al. [10] found the shapes of plagioclase change with supersaturation, i.e. undercooling. The morphology of the plagioclase crystals varies from euhedral tabular crystal to skeletal, to dendritic, and finally to spherulitic as the supersaturation is increased. In analogy, our large plate-shaped graphite of a five minute sample at zone $\mathrm{C}$ also undergo little supersaturation during the solidification of nickel, while the spherical graphite particles at zone A undergo a large supersaturation.

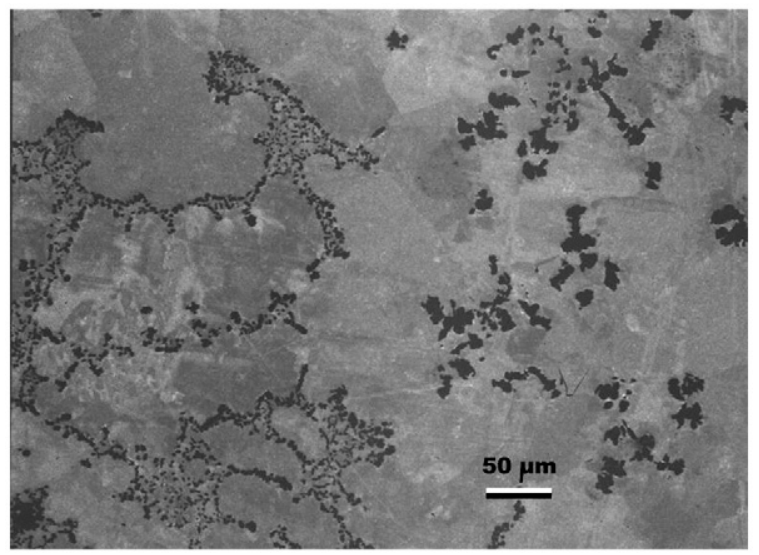

Fig. 4. SEM image of a solidified 5 min arc-discharge nickel block. The picture shows a transition from zone A on the left to zone B on the right. The morphology and sizes are clearly different for the graphite particles in these two zones. 


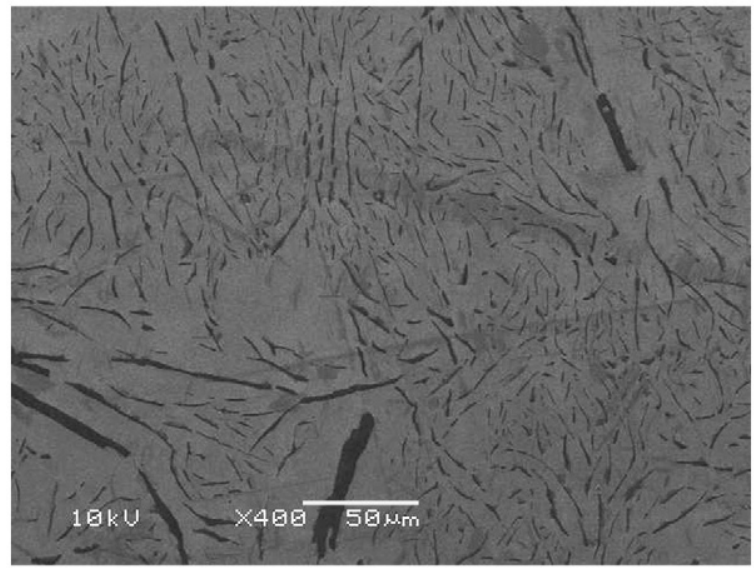

Fig. 5. SEM image shows a zone A cross-section of a nickel block after more than $10 \mathrm{~min}$ arc-discharge and cooling. All the spherical graphite particles can no longer be seen.

Because the temperature and solubility are high, the crystallization and growth of graphite at zone A may be transport-controlled, and therefore the particles become spherical. While at zone $C$, because the temperature and solubility are lower, it may be surface-controlled, and thus the particles become euhedral shape, i.e. platy shaped [11-12].

This work is a preliminary study and further analyses are necessary in order to answer several remaining questions. For example, what is the internal structure of the spherical-like microcrystalline graphite particles (Fig. 7). If the particles are formed by radiated solid crystals, then it indicates that transport-controlled crystallization and growth signifies the most important mechanism. However, if the particles are formed by many nanocrystalline graphitic sheets, then the grain growth of nickel also plays an important role. During the grain growth, the growing nickel grains may push numerous nanocrystalline graphitic sheets along the surface of each grain until they clash with each other. The clashing may create shear forces between the growing grains and "roll" the small graphitic sheets into many ball-like particles.

The key to the success of this experiment is the use of an arcdischarge setup, which provides a unidirectional heating and creates a clear temperature gradient in the nickel during the experiment.

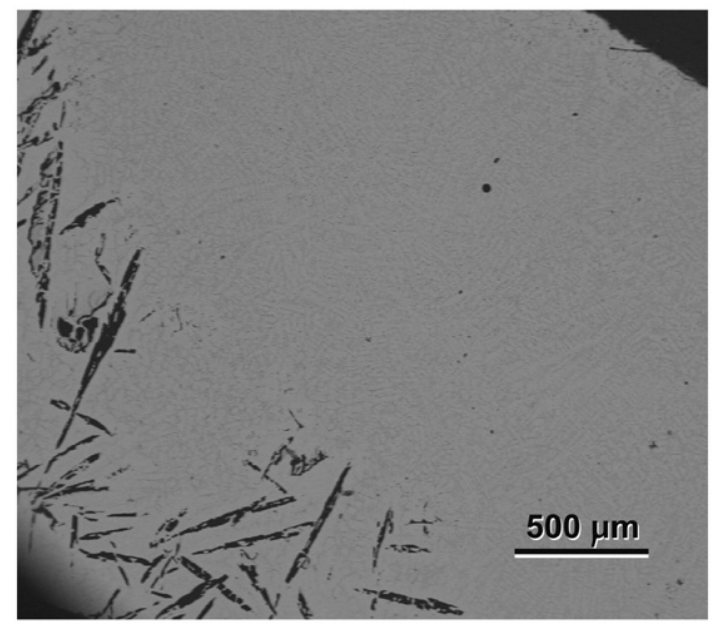

Fig. 6. Cross-section of a nickel sample over $2 \mathrm{~h}$ arc-discharge. Upper right corner is the top and lower left corner is the bottom. Note that in zone A one can hardly find any graphite, and in zone $C$ near the bottom many large graphite plates can be seen

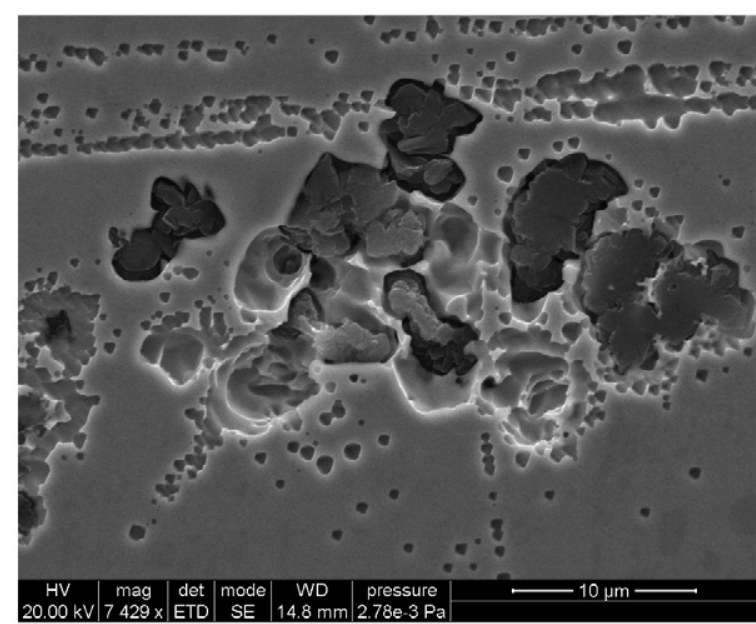

Fig. 7. High magnification SEM image of a number of microcrystalline "spherical" graphite particles found at the hotter zone A (see Fig. 2) in a solidified 5 min arcdischarge nickel block. The pores and cavities were due to the etching of nickel during sample preparation.

Furthermore, this work also demonstrates that it is possible to derive quantitative kinetic data on the phase transformation from diamond to graphite in a catalytic metal, such as $\mathrm{Ni}, \mathrm{Co}$, and $\mathrm{Fe}$.

\section{Conclusions}

By a designed arc-discharge setup, diamond particles easily dissolve in melted nickel. Surprisingly, in the solidified nickel many microcrystalline spherical graphite particles are found at the hotter area (zone A), but not at the cooler area (zone C) where only large platy graphite are found. The forming mechanism of these spherical graphite particles may be due the temperature and solubility at zone A being high; thus the crystallization and growth of graphite is transport-controlled, and therefore the shape of the particles is spherical. In a longer arc-discharge sample, however, almost all the carbon gathers at zone $\mathrm{C}$, and no visible graphite can be found in zone A.

\section{Acknowledgement}

This work was supported in part by the National Science Council, Taiwan, Grant NSC96-2116-M-002-009.

\section{References}

[1] L.J.E. Hofer, E. Sterling, J.T. McCartney, J. Phys. Chem. (1955) 153.

[2] T.J. Konno, R. Sinclair, Acta Metall. Mater. 42 (1994) 1231.

[3] M.H. Teng, S.W. Tsai, C.I. Hsiao, Y.D. Chen, J. Alloys Compd. 434-435 (2007) 678.

[4] C.I. Hsiao, National Taiean University Master Thesis (2006).

[5] V.P. Dravid, J.J. Host, M.H. Teng, B.R. Elliott, J.-H. Hwang, D.L. Johnson, T.O. Mason, J.R. Weertman, Nature 374 (1995) 602.

[6] J.J. Host, M.H. Teng, B.R. Elliott, J.-H. Hwang, T.O. Mason, J.R. Weertman, D.L. Johnson, V.P. Dravid, J. Mater. Res. 12 (1997) 1268.

[7] J.J. Host, V.P. Dravid, M.H. Teng, J. Mater. Res. 13 (1998) 2547.

[8] R.E. Hummel, Understanding Materials Science-History, Properties, Application, 2nd Ed.Springer-Verlag, New York, 2004.

[9] J.-D. Kim, J.-K. Ji, J. Mater. Process. Technol. 176 (2006) 19.

[10] G.E. Lofgren, C.H. Donaldson, R.J. Williams, O. Mullins, T.M. Usselman, Proceedings of the 5th Lunar Scientific Conference, 1974, p. 549.

[11] R.A. Berner, Am. J. Sci. 278 (1978) 1235.

[12] A.C. Lasaga, Kinetic Theory in the Earth Sciences. Princeton Series in Geochemistry. Princeton University Press, Princeton, New Jersey, 1998. 